\title{
QUALIDADE DE VIDA E NIVEL DE ATIVIDADE FÍSICA DE INDIVÍDUOS FREQUENTADORES DE ACADEMIA
}

\section{Quality of life and Physical Activity Level in Individuals who Attend Academy}

\author{
Túlio Brandão Xavier Rocha'; Davi M. A. Prates ${ }^{2}$; Guanis de Barros Vilela Júnior ${ }^{1,2}$; Luciane Manzatto1 ${ }^{1}$ \\ 'UNIMEP - Piracicaba - São Paulo - Brasil \\ ${ }^{2}$ METROCAMP - Campinas - São Paulo - Brasil
}

\begin{abstract}
Resumo: O presente estudo teve como objetivo avaliar a qualidade de vida e o nivel de atividade fisica de um grupo de individuos freqüentadores de uma academia na cidade de Campinas-SP. A amostra foi constituida por 30 individuos, sendo eles 15 homens e 15 mulheres com idade de 20 a 40 anos. A qualidade de vida foi mensurada através do protocolo WHOQOL-bref e o nivel de atividade física através do IPAQ. Os resultados obtidos questionário WHOQOL-bref demonstram valores satisfatórios nos quatros dominios do instrumento. Em relação ao domínio fisico observamos uma média de $82,98 \%$, nos dominios psicológico e de relaçōes sociais, $78,89 \%$, e no domínio meio ambiente a média foi de $67,50 \%$. O nivel de atividade fisica apresentou $30 \%$ nas atividades moderadas e $70 \%$ nas atividades de intensidade elevado. Para os individuos freqüentadores de academia a qualidade de vida e o nivel de atividade demonstraram resultados satisfatórios com valores significativos para o grupo mostrando que através da pratica regular de atividades fisicas pode-se obter uma melhora da qualidade de vida e saúde.

Palavras-chave: Saúde, qualidade de vida e atividade fisica.
\end{abstract}

Abstract: This study aimed to evaluate the quality of life and physical activity level of a group of practitioners of physical activities in a gym in Campinas-SP. The sample consisted of 30 individuals, among them 15 men and 15 women aged 20 to 40 years. Quality of life was measured using the WHOQOL-BRIEF protocol and level of physical activity by IPAQ. The tabulation and data analysis were performed using the program Microsoft Excel and SPSS version 13. In the WHOQOL-BRIEF, we find satisfactory values in the four areas of the instrument. In relation to domain 1 (physical) found an average of 82.98 percent (note that this score is 0 to 100 so the farther from 100 worse quality of life) in domain 2 and 3 (psychological social relations ) 78.89 , and the fourth domain (environment), the average was 67.50 . The level of physical activity gained $30 \%$ in moderate activities, and $70 \%$ in high level activities. For individuals who attend academy, quality of life and activity level had satisfactory results, where this were quite significant for the group, showing that through the practice of regular physical activity it improves quality of life and health in practitioners.

Keywords: Health, quality of life, physical activity.

Aceito em 24/01/2011 - Revista de Educação Física 2011 Abr; 151:32-39. Rio de Janeiro - Brasil

\section{INTRODUÇÃO}

A atividade física é um importante indicador de saúde e é vista como agente preventivo de várias condições como a obesidade, hipertensão arterial, diabetes, além de promover beneficios psiquicos e sociais ${ }^{(1)}$.

O sedentarismo, atualmente, é considerado uma epidemia mundial que acomete cerca de $70 \%$ da população do planeta, e é considerado pela Organização Mundial de Saúde (OMS) o maior inimigo da saúde publica, associado a dois milhões de mortes ao ano globalmente, e por $75 \%$ por mortes nas Américas ${ }^{(2)}$.

Com esses dados observamos que os individuos da sociedade moderna vêm cada vez menos praticando atividades físicas. As barreiras que impedem os individuos de se engajarem em uma prática regular de atividades físicas são inúmeras: falta de conhecimento sobre os beneficios da atividade fisica, motivos como falta de tempo e equipamentos, pouco ou nenhum incentivo, falta de dinheiro, poucas opções de espaços para a realização de atividades, entre outros. Por outro lado, alguns fatores favorecem a prática regular de atividades físicas nas academias, tais como a busca pela melhora ou manutenção da saúde, motivos estéticos ou envolvimento social.

Nesse contexto, a saúde tem sido definida ampliando-se o conceito da OMS - um estado de completo bem estar físico, mental e social, não apenas a ausência de doenças - mas como parte 
prioritária de bens básicos necessários para que o individuo desenvolva o seu potencial no ser e faze $^{r(3)}$. Assim, a questão de saúde envolve muito mais do que a não doença, envolve outros aspectos e características que o ser humano pode apresentar, afastando os fatores de risco e doenças.

Autores como Guedes (1997) e Barbanti (1986), investigaram a saúde da população de maneira geral, e estabeleceram que a hipocinesia (ausência de movimento), também está ligada a falta de saúde ${ }^{(4.5)}$

A partir da carta de Ottawa, em 1986, a saúde passou a ser "construída pelo cuidado de cada um consigo mesmo e com os outros, pela capacidade de tomar decisões e de ter controle sobre as circunstâncias da própria vida e pela luta para que a sociedade ofereça condições que permitam a obtenção da saúde por todos os seus membros" (6). Além disso, a saúde tem determinantes sociais, que podem ser entendidos como "fatores sociais, econômicos, culturais, étnico-raciais, psicológicos e comportamentais que influenciam a ocorrência de problemas de saúde e seus fatores de risco na população"(7).

Reforçando esta idéia, Malina (2002), cita que a atividade física regular é frequentemente vista como necessária para o crescimento. Isso demonstra-nos que o exercício físico, junto com as condições socioeconômicas de higiene e meio ambiente é considerado uma influencia ambiental para o crescimento, sendo importante desde a fase infantil ${ }^{(8)}$

Além de todos esses aspectos relacionados à saúde, não podemos esquecer-nos da influencia dela para se ter uma boa Qualidade de Vida (QV).

A noção de QV transita em um campo semântico e polissêmico: de um lado, está relacionada ao modo, condições e estilo de vida, de outro, inclui as idéias de construção humana e valores socialmente constituidos, e por fim, relaciona-se ao campo da democracia, do desenvolvimento e dos direitos humanos e sociais. No que concerne à saúde, as noções se unem em uma resultante social da construção coletiva dos padrões de conforto e tolerância que determinada sociedade estabelece como parâmetros para $\mathrm{si}^{(9,10)}$.
De acordo com Vilela Júnior e Leite (2008), o termo Qualidade de Vida é tratado sobre diversos olhares, seja da ciência, senso comum, ponto de vista objetivo ou subjetivo e em abordagens individuais e coletivas ${ }^{(11)}$.

Conforme Minayo et al (2005), QV è uma noção eminentemente humana que aproxima-se do grau de satisfação encontrado na vida familiar, amorosa, social e ambiental. A autora pressupõe uma sintese cultural de todos os elementos que determinada sociedade considera como seu padrão de conforto e bem-estar (12).

Podemos citar que na visão mais voltada para saúde, QV, está na capacidade de viver sem doenças, superando dificuldades dos estados ou condições de morbidade.

Com isso, o assunto qualidade de vida, vem sendo bastante recorrente nos dias atuais, o que torna imprescindiveis pesquisas referentes a esse tema.

Neste sentido, compreender o constructo QV é apreendê-la enquanto processo histórico de nossa psique, de nossa vida em sociedade e de como nos relacionamos com o ambiente em que vivemos. Assim sendo, QV é a mediação que o individuo faz entre aquilo que tem e aquilo que é; na perspectiva sociocultural e biopsiquica. A atividade física regular e orientada é capaz de meIhorar a saúde das pessoas e assim promover a melhoria da QV e da autonomia para a cidadania.

Parece que a busca pelo significado do termo qualidade de vida seja tão antiga quanto à civilização, sendo que diferentes referenciais filosóficos conceituam o que seja viver com qualidade. Segundo a visão aristotélica, a vida com qualidade referia-se aos sentimentos relacionados à felicidade, realização e plenitude. Através da OMS o termo passou a ser utilizado, quando este órgão redefiniu o conceito saúde e incorporou a noção de bem-estar físico, emocional e social, desencadeando uma discussão a respeito da possibilidade de medir o bem-estar ${ }^{(13)}$

[...] o avanço tecnológico que propiciou maiores possibilidades para a recuperação da saúde e prolongamento da vida; o segundo fator consiste na mudança do panorama epidemiológico das doenças, sendo que o perfil dominante passou a 
ser o das doenças crônicas; o terceiro fator trata da tendência de mudança sobre a visão do ser humano, antes visto como organismo biológico que deveria ser reparado e hoje, como um agente social (DINIZ; SCHOR, 2005, p.03).

Portanto, no contexto deste trabalho, compreendemos a QV como resultado da mediação daquilo que temos e aquilo que somos.

Outro dado importante para esta pesquisa é enfatizar os métodos de avaliação da atividade física, antes de definir qualidade de vida e o nível de atividade. É necessário encontrar meios para quantificar de maneira eficiente e econômica o nível de atividade física da população. Os questionários são formas viáveis e econômicas, embora seja discutível a fidedignidade dessas medidas. No Brasil, especificamente, os programas de educação (atividade) física têm início alicerçados em bases médicas, procurando formar 0 indivíduo "saudável" com uma boa postura e aparência física.

A mensuração do nível de atividade física em grandes grupos populacionais requer instrumentos de fácil aplicação, boa precisão e de baixo custo. $O$ instrumento a ser utilizado para medir a prática de atividade física será o Questionário Internacional de Atividades Físicas - IPAQ - versão longa, proposto pela OMS ${ }^{(14)}$.

O IPAQ classifica como atividades moderadas aquelas que "precisam de algum esforço físico e que fazem respirar um pouco mais forte do que o normal", enquanto que as atividades vigorosas "precisam de um grande esforço físico e que fazem respirar muito mais forte do que o normal.

Outro teste, o WHOQOL-Bref é um instrumento genérico de qualidade de vida composto de 26 itens pertinentes à avaliação subjetiva do individuo em relação aos aspectos que interferem em sua vida. A versão em português utilizada neste estudo foi validada por Fleck et al. no ano de 2000(15). Por tratar-se de um construto multidimensional, este instrumento de medida da qualidade de vida abrange quatro domínios - físicos, psicológico, relações sociais e meio ambientes. A avaliação da QV será obtida através do instrumento da escala abreviada de Qualidade de Vida da Organização Mundial de Saúde (WHOQOL-
Bref) sendo a mesma usada em diversos estudos científicos no pais.

Após citarmos formas de quantificar e qualificar atividade física, enfatizamos que o estilo de vida da população modificou-se nas últimas décadas, acarretando um aumento do número de pessoas sedentárias, principalmente em paises industrializados.

Outra doença que não podemos deixar de comentar é o acidente vascular cerebral (AVC), que continua sendo uma das grandes preocupações da atualidade, tendo em vista, essa ser a terceira maior causa de morte por doença no mundo, perdendo apenas para as doenças cardiacas e o câncer ${ }^{(16)}$.

Assim, sabe-se que a atividade física regular, melhora a aptidão física através de programas de exercícios físicos, incluindo os praticados em academias, ocasionando uma melhora nas capacidades físicas, onde os exercícios sistematizados favorecem uma melhora na qualidade de vida dos praticantes ${ }^{(17)}$.

Tratando-se de atividades em academia, citaremos a musculação, com um exemplo de prática possivel, visto que, essa é uma atividade que, atualmente, passou a ser muito praticada, principalmente devido o grande número de estabelecimentos que oferecem esses serviços, facilitando o acesso a ela. Sua prática, tem contribuido para melhora da qualidade de vida da população, que orientada de forma planejada, seguindo rigorosamente alguns critérios, pode ser prescrita para grupos bem heterogêneos, incluindo, pessoas de diferentes idades, sedentários, portadores de necessidades especiais e pessoas com as mais variadas doenças, levando-se em conta ainda a necessidade de um programa diferenciado e personalizado individualmente, para contribuir de maneira positiva, tanto na diminuição das sintomatologias, dos fatores de risco associados a cada doença, ou mesmo para quem pratica buscando $Q V$, não tento limitações ${ }^{(18) .}$

Segundo Ravagnani, Cunha Junior, Werk e Coelho (2007) a prática regular de atividades fisicas traz vários benefícios à saúde, entre eles: aumento do gasto energético, controle da gordura corporal, melhora dos perfis lipídicos e glicêmicos, 
redução da pressão arterial, melhora da coordenação motora, da força muscular, do equilibrio, da flexibilidade e das capacidades cardiorrespiratória, além dos ganhos psicológicos, como redução do quadro de depressão e isolamento social e aumento da auto-estima(19).

Evidentemente muitas variáveis devem ser observadas quando se trata de atividade física. De acordo com Pieron (2004), além da duração e freqüência da atividade, a intensidade do esforço também desempenha um papel importante induzindo efeitos de aptidão. As recomendações de especialistas da saúde e epidemiologistas mudaram a ênfase no modelo de intensidade relativamente alta, propondo, em vez disso, recomendações para que se faça a atividade a uma intensidade moderada $^{(20)}$.

Estudos epidemiológicos colocaram em evidencia uma razão inversa entre o nivel de atividade fisica habitual e o risco de acidente cardiovascular. Em outras palavras: quanto menor é o nivel de atividade física, maior é o risco cardiovascular ${ }^{(20)}$.

Outra constatação importante sobre o efeito da atividade física vem de estudos que têm demonstrado uma relação inversa entre os niveis de atividade física e a mortalidade por todas as causas ${ }^{(21}$. 22. 23). Sabe-se que problemas de ordem emocional, como depressão e angústias, têm associação inversa com a prática de atividades físicas ${ }^{(24,25,26)}$.

Malta et al em 2006, afirmou que dados da Organização Mundial de Saúde de 2002 indicam que $22 \%$ das doenças cardíacas, 10 a $16 \%$ dos casos de diabetes tipo 2 e de cânceres de mama, cólon e reto poderiam ser evitados com a realização de um volume suficiente de atividade fisica ${ }^{(27)}$.

De acordo com Batista et al (2003), evidências baseadas em estudos epidemiológicos confirmaram o papel decisivo da prática da atividade física na promoção da saúde, na qualidade de vida e na prevenção e controle de diversas doenças. Diretrizes para a promoção de estilos de vida saudáveis têm sido recomendadas por órgãos envolvidos com a saúde pública(28).

Segundo autores como Silva, Nahas (2004) políticas públicas de saúde devem incentivar a adoção de um estilo de vida ativo visando melhorias na qualidade de vida das pessoas de todas as idades, principalmente nas idosas, onde os riscos da inatividade física se potencializam, diminuindo precocemente os anos de vida útil(29).

O objetivo desta pesquisa foi avaliar a qualidade de vida e o nivel de atividade física em indivíduos que frequentam uma academia na cidade de Campinas através do Woqol-bref e IPAQ.

\section{MATERIAL E MÉTODOS}

Estudo transversal descritivo cuja amostra foi composta por um grupo de 30 indivíduos dentre estes 15 homens e 15 mulheres, com idade entre 20 e 40 anos. Todos os individuos foram informados sobre a proposta do estudo e os procedimentos aos quais seriam submetidos e assinaram termo de consentimento livre e esclarecido.

$\mathrm{O}$ grupo era formado por individuos praticantes de musculação há pelo menos 1 ano sendo eles profissionais autônomos e funcionários de diferentes áreas de atuação.

\section{Procedimento da coleta de dados}

Foi utilizado e aplicado o questionário internacional de atividades físicas para a avaliação do nivel de atividades físicas, IPAQ, e também o questionário WHOQOL-Bref, que tem por finalidade saber o que o individuo sente a respeito de sua qualidade de vida, saúde e outras áreas de sua vida.

Os individuos responderam aos dois questionários sem auxilio; as perguntas do WHOQOL se referiam às duas semanas recentes em relação à percepção da qualidade de vida, e o IPAQ se referia a uma semana habitual. O tempo gasto para as respostas do questionário foi em média de dez minutos.

\section{Tratamento de dados}

A análise dos dados foi realizada nos softwares Microsoft Excel®e SPSS $₫$ versão 17.0 através da sintaxe do WHOQOL-bref e do IPAQ, além da estatística descritiva e correlações de Spearman entre os dominios e do dominio físico com o IPAQ. A consistência interna das respostas foi verificada através da determinação do coeficiente alfa de Cronbach. 


\section{RESULTADOS E DISCUSSÃO}

O coeficiente de Cronbach foi de 0,78 o que atesta boa consistência interna das respostas obtidas, entretanto, as questões de número 26 (relativa à necessidade de medicamentos) e 4 (relativo à estados de humor, ansiedade, etc) foram as que mais comprometeram o valor deste coeficiente. Os resultados do WHOQOL-bref são apresentados na tabela a seguir.

\begin{tabular}{cccc}
\hline $\begin{array}{c}\text { MEDIA D1 } \\
\text { Dominio Fisico }\end{array}$ & $\begin{array}{c}\text { MEDIA D2 } \\
\text { Psicológico }\end{array}$ & $\begin{array}{c}\text { MEDIA D3 } \\
\text { Social }\end{array}$ & $\begin{array}{c}\text { MEDIA D4 } \\
\text { Meio Ambiente }\end{array}$ \\
\hline 82,98 & 78,89 & 78,89 & 67,50 \\
\hline
\end{tabular}

TABELA 1 - Resultados de cada domínio do Whoqol breve

A melhor pontuação obtida no dominio físico (D1) provavelmente tem relação com o fato de os individuos entrevistados já estarem habituados à prática de atividades fisicas e, possivelmente, devem ter ciência dos benefícios que ela proporciona. Os dominios psicológico (D2) e de relações sociais (D3) alcançaram escores menores, mas não menos expressivos, fato este que poderia ser explicado por benefícios como melhora da autoestima, autoconceito e da imagem corporal, e pelas relações interpessoais encontradas no ambiente da academia, com amigos, professores e funcionários. Entretanto, o domínio meio ambiente (D4) foi o que apresentou menor porcentagem, o que pode ser reflexo da precariedade dos serviços e oportunidades, por exemplo no que se refere a espaços para lazer ativo, segurança pública, transporte e condições de moradia.

Foram encontradas correlações significativas $(p<0,01)$ entre D1 e D2, D1 e D4, e D3 e D4. Correlação significativa a 0,05 foi encontrada entre D2 e D3.

Podemos refletir sobre a correlação entre o domínio físico e psicológico tendo em vista a colaboração da atividade física sobre a percepção de bem estar do individuo. As correlações entre os do- minios físico e de relações sociais com o dominio meio ambiente sugere que as condições de infraestrutura são satisfatórias para aquela população e que, possivelmente, as relações interpessoais estabelecidas por estas pessoas estão condicionadas aos ambientes frequentados. De certo modo, o bem estar social está mais relacionadas ao estado psicológico que à atividade física propriamente dita, e parece não haver influência negativa do meio ambiente sobre o dominio psicológico.

Ainda com relação ao domínio 4, outro estudo Cerchiari (2004), realizado com 558 universitários, relacionando saúde mental e qualidade de vida, demonstraram também um baixo escore com relação ao meio ambiente, quando comparado aos outros dominios, embora para essa pesquisa todos os dominios tiveram resultados inferiores(30).

Vilela Júnior et. al (2010) deixa claro o impacto negativo das questões relacionadas ao meio ambiente para a QV em idosas praticantes de atividades físicas. Infraestrutura, transporte, espaço para lazer ativo, condições de moradia e saúde são fatores que prejudicam a $\mathrm{QV}^{(31)}$.

Custodio, et al, 2004, avaliaram o nivel da qualidade de vida em estudantes de psicologia e nutrição e concluiram que problemas com segurança e questão financeiras influenciaram no resultado inferior no D4, em concordância com este estudo ${ }^{(32)}$.

Zamarim, et al. (2006) compararam a percepção de QV, através do Whoqol, em individuos freqüentadores e não freqüentadores de academia. $\mathrm{O}$ estudo demonstrou não haver diferenças diferença entre os grupos ${ }^{(33)}$.

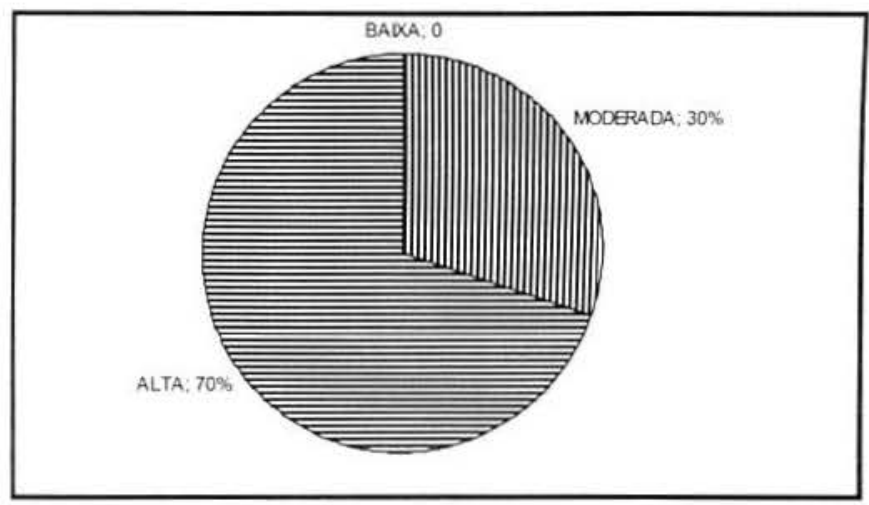

GRÁFICO 1 - Resultado do nivel de atividade fisica 
De acordo com os resultados do WHOQOL, percebe-se que a prática de atividade física é um fator determinante da QV para esta população. Conforme os resultados do IPAQ pode ser verificado que o nivel de atividade física de alta intensidade é bastante satisfatório e os valores para atividade moderada também é expressivo.

A correlação entre o dominio físico do Whoqol e o resultado do IPAQ não foi significativa $(p=0,450)$. Isto possibilita supor a não existência de uma relação causal entre o nível de atividade física e a percepção de qualidade de vida.

O termo "atividade física" se refere a qualquer movimento, produzido por músculos esqueléticos, que resulta em gasto energético maior que os niveis de repouso. Por outro lado, "exercício físico", o que se presume praticar em academia, é uma atividade física planejada, estruturada e repetitiva, que tem como objetivo melhorar ou manter a saúde ou aptidão fisica ${ }^{(34)}$. Portanto não se pode ter certeza de que o nivel de atividade física se deve às práticas na academia, uma vez que movimentos no trabalho, lazer em afazeres domésticos tem influência sobre os resultados do IPAQ. O fato de todos os voluntários frequentarem a academia regularmente $e$ serem praticantes de musculação, pode ter influenciado os resultados do IPAQ, embora $30 \%$ tenha tido resultado apenas moderado.

Com relação ao estudo desenvolvido por Almeida, et al (2010), que pesquisou 30 praticantes de musculação, com idade entre 17 e 32 anos, avaliando o nivel de satisfação e qualidade de vida desses, mostrou que $80 \%$ dos voluntários afirmaram perceber uma melhora significativa na qualidade de vida, com a prática da musculação e $6,6 \%$ disseram perceber uma melhora razoável e apenas $13,3 \%$ responderam não ter notado nenhuma diferença ${ }^{(35)}$.

\section{CONCLUSÃO}

Para os individuos freqüentadores de academia a qualidade de vida e o nivel de atividade tiveram resultados satisfatórios nas questões sobre saúde e nivel de atividade física, em que o grupo avaliado apresentou respostas relevan- tes aos domínios físicos como relações sociais, ambiente, psicológica, e física. Nota-se que o domínio físico teve resultado bastante significativos para o grupo, acompanhado de resultados expressivos do IPAQ, apesar de não apresentarem correlação, mostrando que a pratica regular de atividades físicas oferece uma grande contribuição na melhora da qualidade de vida e saúde. Como este estudo pode-se concluir que individuos frequentadores de academia, realizando atividades físicas têm uma vida mais satisfatória nos critérios de bem estar social e físico.

Dada a complexidade do tema da percepção da qualidade de vida este estudo apresenta limitações relativas ao número de sujeitos avaliados e ao provável viés na pontuação do domínio físico, posto que existe a possibilidade de que frequentadores de uma academia possam super estimar os beneficios da atividade física em sua qualidade de vida.

\section{Conflitos de interesse}

Os autores declaram não haver competição ou conflitos de interesse.

\section{REFERÊNCIAS BIBLIOGRÁFICAS}

1 - Morrato, Elaine H. et al. Physical Activity in U.S. Adults With Diabetes and At Risk for Developing Diabetes, 2003. In. Diabetes Care, fev 2007; 30(2): 2039. Disponivel em: http://care.diabetesjournals.org/content/30/2/203.full.pdf+html (19 mar. 2010).

2 - Pozena, Regina; CUNHA, Nancy F. S.: Projeto "Construindo um Futuro Saudável através da prática da atividade física diária". Saúde e Sociedade, 2009: 18(1) . Disponivel em: http://www.scielo.br/pdf/sausoc/ v18s1/09.pdf (03 abr. 2010).

3 - Arango, Alba L. V. Nuevas dimensiones del concepto de salud: el derecho a la salud em el estado social de derecho - Hacia la Promoción de la Salud, 2007; 12: 63 - 78. Disponivel em: http://www.scielo.org.co/pdf/ hpsal/v12n1/v12n1a05.pdf (19 mar 2010)

4 - Guedes, Dartagnan. P.; GUEDES, Joana. E. R. P. Crescimento, Composição Corporal e Desempenho Motor de Crianças e Adolescentes. São Paulo: Balieiro, 1997 
5 - Barbanti, Valdir. J. Aptidão Fisica: Conceitos e Avaliações. Revista Paulista de Educação Fisica, 1986; 1(1): 24-32 .

6 - Buss Heidmann, Ivone T.S.; Almeida, Maria Cecilia P.; BOEHS, Astrid E.; WOSNY, Antônio M.; MONTIDELLI, Marisa. Promoção À Saúde: trajetória histórica de suas concepções - Texto Contexto Enferm, Florianópolis, Abr-Jun, 2006; 15(2): 352-8. Disponivel em: http://www.scielo.br/pdf/tce/v15n2/a20v15n2.pdf mar. 2010).

7 - Buss, Paulo N.; Pllegrini filho, Alberto. Iniqüidades em saúde no brasil, nossa mais grave doença: comentários sobre o documento de referência e os trabalhos da comissão nacional sobre determinantes sociais da saúde. Cad. Saúde Pública, 2006; 22(9),Dissponivel em: http://www.scielo.br/scielo.php?pid=\$0102$311 \times 2006000900033 \&$ script $=$ sci_arttext\&tIng $=e n \quad(20$ mar. 2010).

8 - Malina, Robert. M.; BOUCHARD, Claude. Atividade Fisica do Atleta Jovem: Do Crescimento à Maturação. São Paulo: Roca, 2002.

9 - Almeida, Marco A. B.; VITAGLIANO, Luis Fernando. Patologias sociais e a qualidade de vida na sociedade moderna. Revista Brasileira de Qualidade de Vida, Ponta Grossa, 2009; 1(2): 1-7, Disponível em: http://www. boletimef.org/biblioteca/2660/Patologias-sociais-e-aqualidade-de-vida-na-sociedade-moderna (4 abr. 2010)

10 - Gutierrez, M. Perfil descriptivo-situacional del sector de la promoción y educación en salud: Colombia. In Aroyo Hv E Cerqueira Mt (Eds.), 1996. La Promoción De La Salud Y La Educación Para La Salud Em America Latina: Un Analisis Sectorial. Editorial De La Universidad De Puerto Rico, 144.

11 - Vilela Júnior, Guanis. B.; Leite, Neiva. Qualidade de Vida e Saúde: Avaliação pelo QVS-80. In: Vilarta, R.; Gutierrez, G.L.; Carvalho, T.H.P.F.; Gonçalves, A. Qualidade de vida e novas tecnologias. Campinas: Ipês Editorial, , cap 7, p. 129-138, . Disponivel em: http:// www.fef.unicamp.br/departamentos/deafa/qvaf/livros/ foruns_interdisciplinares_saude/tecnologia/tecnologia. pdf (04 abr. 2010)

12 - MINAYO, Maria Cecilia S. A saúde em estado de choque. Rio de Janeiro: Espaço E Tempo, 1992.

13 - Diniz, D.P.; Schor, N., 2005, p.1 apud CHEPP, C. C. Estudo transversal da qualidade de vida através da escala whoqol-bref da população octogenária e nonagenária de siderópolis, Universidade do Extremo Sul Catarinense curso de Medicina, 2006.
14 - Pardini, R.; Matsudo, S. M.; Araújo, T.; Matsudo, V.; Andrade, E.; Braggion, G. et al. Validação do questionário internacional de nivel de atividade fisica (Ipaq - Versão 6): Estudo Piloto em Adultos Jovens Brasileiros. Rev. Bras. Ciên.. e Mov., 2001; 9(3): 45-51. Disponivel em: http://www.ucb.br/mestradoef/RBCM/9/9\%20 -\%203/completo/c_9_3_6.pdf (04 abr. 2010)

15 - Fleck, Marcelo P. A.; LOUZADA, Sérgio.; XAVIER, Marta.; CHACHAMOVICH, Eduardo; VIEIRA, Guilherme; SANTOS, Lyssandra; PYNSON, Vanessa. Aplicação da versão em português do instrumento abreviado de avaliação da qualidade de vida "WHOQOL-bref". Revista de Saúde Pública, 2000; 34(2): 178-83. Disponivel em: http://www.scielo.br/pdf/rsp/v34n2/1954.pdf (2 abr. 2010).

16 - Costa, Alerto M.; DUARTE, Edison. Atividade fisica e a relação com a qualidade de vida, de pessoas com seqüelas de acidente vascular cerebral isquêmico (AVCl). Rev. Bras. Ciên. e Mov, 2002; 10 (1): 4754. Disponivel em: http://www.ucb.br/Mestradoef/ RBCM/10/10\%20-\%201/r7v10n1.pdf (04 abr 2010).

17 - Pitanga, Francisco. J. G. Epidemiologia, atividade fisica e saúde.

Rev. Bras. Ciên. e Mov. Brasilia, 2002; 10(3). Disponível em: http://www.ucb.br/mestradoef/RBCM/10/10\%20 -\%203/c_10_3_8.pdf (04 abr. 2010).

18 - Campos, Mauricio de Arruda. Musculação: diabéticos, osteoporóticos, idosos, crianças, obesos/ Rio de Janeiro: $4^{\mathrm{a}}$ edição: Sprint ,2008.

19 - Ravagnani, Fabricio C. P.; CUNHA JÚNIOR, Arnaldo T.; WERK, Rafael; COELHO, Christianne F.: Composição corporal e objetivos na procura de atividades fisicas supervisionadas entre iniciantes em programa de exercícios físicos em academia de Botucatu-SP. In Fit Perf J, Rio de Janeiro, 2007; 6(3): 151. Disponivel em: http://www.fpjournal.org.br/painel/arquivos/441-3\%20 Exercicios\%20na\%20Academia\%20Rev\%203\%20 -\%202007.pdf (02 abr. 2010).

20 - Pieron, Maurice. Estilo de vida, prática de atividades físicas e esportivas, qualidade de vida. Fitness \& Performance Journal, 2004; 3(1), p.10 -17. Disponivel em: http://dialnet.unirioja.es/servlet/ articulo? codigo $=2953114$ (04 abril 2010).

21 - Haapanen, Nina; Miilunpalo, Seppo, VUORI, Ilkka; OJA, Pekka, PASANEN, Matti. Characteristics of leisure time physical activity associated with decreased risk of premature all-cause and cardiovascular disease mortality in middle-aged men. American Journal of Epidemiology, 1996; 143 (9). Disponivel em: http://aje.oxfordjournals.org/cgi/reprint/143/9/870 (02 abr. 2010). 
22 - Lissner, Lauren; Bengtsson, Calle; Björkelund, Cecilia; Wedel, Hans: Physical activity levels and changes in relation to longevity. American Journal of Epidemiology. 1996; 143(1). Disponivel em: http://aje.oxfordjournals. org/cgi/content/abstract/143/1/54 (03 abr. 2010).

23 - Andersen, Lars B.; Schnohr, Peter; Schroll, Marianne; HEIN, Hans O.: All-Cause Mortality Associated With Physical Activity During Leisure Time, Work, Sports, and Cycling to Work. Arch Intern Med. 2000; 160(11). Disponivel em: http://archinte.ama-assn.org/cgi/content/abstract/160/11/1621 (03 abr. 2010).

24 - Fox, Kenneth R: The influence of physical activity on mental well-being. Public Health Nutrition. 1999; 2(3a). Disponivel em: http://journals.cambridge.org/action/displ ayFulltext?type $=1 \&$ fid $=625660$ jid $=\&$ volumeld $=\&$ issueld $=3 a \&$ aid $=554844 \&$ bodyld $=$ \&membership Number $=\&$ soci etyETOCSession $=(03 \mathrm{abr} .2010)$

25 - Hallal, P. C.; Victora, C. G.; Wells, J. C. K.; Lima, R. C.: Physical Inactivity: Prevalence and Associated Variables. Brazilian Adults. Med. Sci. Sports Exerc., 2003; 35(11). Disponivel em http://journals.luw.com/acsm-msse/Abstract/2003/11000/Physical_Inactivity_Prevalence_and_Associated.18.aspx (03 abril 2010).

26 - Goodwin, Renee D.: Association between physical activity and mental disorders among adults in the United States. Preventive Medicine. 2003; 36(6). Disponivel em: http://www.sciencedirect.com/science? ob=ArticleURL\&_udi=B6WPG-48B5KPX-4\&_user=10\&_ coverDate $=06 \% 2 \mathrm{~F} 30 \% 2 \mathrm{~F} 2003 \&$ rdoc $=1 \&$ f $\mathrm{fmt}=$ high\&_ orig $=$ search \& sort $=d \&$ docanchor $=\&$ view $=c \&$ searchStrld $=1 \overline{27} 99390 \overline{2} 2 \&$ rerunOrigin $=$ scholar. google\&_acct $=C 00005022 \overline{1}$ \&_version $=1 \&$ _urlVersion $=0 \&$ userid $=10 \& \mathrm{md} 5=2 \mathrm{ad} 64862 \mathrm{e} 0 \mathrm{~b} 03 \mathrm{a} 5 \overline{1} 64927 \mathrm{~d}$ ae265328̄91 (03 abr 2010).

27 - Malta, Deborah C.; et al.: Padrão de atividade fisica em adultos brasileiros: resultados de um inquérito por entrevistas telefônicas. Epidemiol. Serv. Saúde. 2006; 18(1). Disponivel em: http://scielo.iec.pa.gov.br/pdf/ess/ v18n1/v18n1a02.pdf (03 abr. 2010).

28 - Batista, Daniela C.; Chiara, Vera I.; Gugelmin, Silvia A.; Martins, Patricia D.. Atividade física e gestação: saúde da gestante não atleta e crescimento fetal, REV. Bras. Saúde Matern. Infant. 2003; 3(2): 151-8. Disponivel em: http://www.scielo.br/pdf/rbsmi/v3n2/a04v03n2.pdf abril 2010).

29 - Silva, Daniela K., Nahas, Markus V. Atividade fisica habitual e qualidade de vida relacionada à saúde em muIheres com doença vascular periférica. R. bras. Ci.e Mov. 2004; 12(4): 63-8. Disponivel em: http://cev.org.br/biblioteca/atividade-fisica-habitual-qualidade-vida-relacionada-saude-mulheres-com-doenca-vascular-periferica (04 abr 2010).
30- Cherciari, E. A. N. Saúde mental e Qualidade de vida em estudantes universitários de campinas. Tese Doutorado. Universidade Estadual de Campinas, Faculdade Ciências Médicas, 2004.

31 - Vilela Júnior, G. B. et al, Qualidade de vida e capacidade de equilibrio durante o processo de envelhecimento de mulheres praticantes de atividade física. Revista Brasileira de Qualidade de Vida. 2010; 2(2): 1825. Disponivel em: http://www.pg.utfpr.edu.br/depog/ periodicos/index.php/rbqv/article/viewFile/664/553 (16 jan. 2011).

32 - Custodio, E. M.; Gomes, M. R. R.; Avoglia, H. R. C.; Bastos, I.. Qualidade de Vida entre Universitárias: Estudos preliminares com o WHOQOL-Bref. Boletim Academia Paulista de Psicologia. 2004; 24(3): 47-57.

33 - Zamarin, M. A.; Miranda, M. L.; Valadi, M.. Influêncida da prática de atividade física em academias na qualidade de vida de adultos universitários: um estudo compartivo. Revista Integração. 2006; 47: 351-356.

34- Cheik, N.C.; Reis, I. T.; Heredia, R. A. G.; Ventura, M. L.; Tufik, S.; Antunes, H. K. M.; Mello, M. T. Efeitos do exercício físico e da atividade fisica na depressão e ansiedade em individuos idosos. R. bras. Ci. e Mov. 2003; 11(3): 45-52.

35 - Almeida, T. F., Souza, P. P. S, Salene, A. P. F., Almeida Júnior, P. L.. Análise do nivel de satisfação e Qualidade de Vida de praticantes de musculação. EFDeportes.com, Revista Digital. 2010; 15(148). Disponivel em: http://www.efdeportes.com/efd148/qualidade-de-vidade-praticantes-de-musculacao.htm (17 jan. 2011)

\footnotetext{
Endereço para correspondência:

Rua Renato Rabelo, 109, Morada do Parque. Montes

Claros - MG - Cep - 39401-364

tuliofisioef@gmail.com

personal_daviprates@hotmail.com

guanis@gmail.com

lu_manzatto@yahoo.com.br
} 